\title{
Two loop four dimensional formulation of dimensionally regularized amplitudes in quantum chromodynamics
}

\author{
Angelo Raffaele Fazio ${ }^{1, *}$ \\ ${ }^{1}$ Departamento de Física, Universidad Nacional de Colombia, Ciudad Universitaria, Bogotá D.C., \\ Colombia
}

\begin{abstract}
We propose a pure four-dimensional formulation (FDF) of the ddimensional regularization of one and two loop scattering amplitudes in quantum chromodynamics. In our formulation particles propagating inside the loops are represented by massive internal states regulating the divergences. The equivalence between the FDF and the Four Dimensional Helicity scheme is discussed. We present explicit representations of the polarization and helicity states of the four-dimensional particles propagating in the loops. They allow for a complete, four-dimensional, unitarity-based construction of d-dimensional amplitudes. Generalized unitarity within the FDF does not require any higherdimensional extension of the Clifford and the spinor algebra.
\end{abstract}

\section{Introduction}

The ultraviolet (UV) and infrared (IR) infinities arising in the higher order perturbative calculations in quantum field theories can be treated by dimensional regularization [1]. Those divergences are usually nested like in the following integral

$$
\int \frac{d^{4} k}{(2 \pi)^{4}} \frac{1}{\left(k^{2}\right)^{2}}=\pi^{2} \int_{0}^{\infty} d k^{2} \frac{1}{k^{2}}=\pi^{2} \int_{0}^{\infty} \frac{d x}{x}
$$

which is UV divergent for $k^{2} \rightarrow \infty$ and IR divergent for $k^{2} \rightarrow 0$. Dimensional regularization is based on the continuum dimension method by first generalizing the dimensionality of the space from four to $d$ integer, such that the integral converges, and then replacing $d$ by the complex number $4-2 \epsilon$, which contains the regularizing parameter $\epsilon$. Symbolically

$$
\int \frac{d^{4} k}{(2 \pi)^{4}} \rightarrow \mu_{\mathrm{DS}}^{4-d} \int \frac{d^{d} k}{(2 \pi)^{d}}
$$

with $\mu_{D S}$ the unit mass needed to take into account the change of the engineering dimensions of the couplings of the theory. The $d$-dimensional integral in (1) can be treated as

$$
\int d^{d} k \frac{1}{\left(k^{2}\right)^{2}}=\frac{\pi^{\frac{d}{2}}}{\Gamma\left(\frac{d}{2}\right)} \int_{0}^{\infty} d k^{2}\left(k^{2}\right)^{\frac{d}{2}-3} .
$$

The continuation from 4 to $d=4-2 \epsilon$ dimensions makes all momentum integrals well defined and UV and IR singularities appear in the Laurent expansion of meromorphic functions as $\frac{1}{\epsilon^{n}}$

*e-mail: arfazio@unal.edu.co 
poles. Actually the integral (1), combined with other UV and IR divergent terms, is usually defined [2] so that to cancel those divergences and not to bring contributions to the finite part.

Consider the physical cross section at the Next-to-Leading Order which receives by the Kinoshita- Lee- Nauenberg theorem the real and virtual contributions

$$
\sigma=\sigma_{V}+\sigma_{R}=\int d \Phi_{V}\left|M_{V}\right|^{2}+\int d \Phi_{R}\left|M_{R}\right|^{2}
$$

identified by the corresponding amplitudes $M_{R}$ and $M_{V}$. A complete dimensional scheme $(D S)$, meaning a complete procedure to make the analytical dimensional continuation in the dynamics of the theory is reflected at the level of the Feynman integrals by the way of treating the gauge boson metric, generically indicated in the following by $g$ :

$$
\begin{gathered}
\sigma^{D S}=\underbrace{\int d \Phi_{V}\left|M_{V}(\ldots .,[g], \ldots . .)\right|^{2}}_{\frac{a}{\epsilon^{2}}+\frac{b}{\epsilon}+c+d \epsilon+e \epsilon^{2}+\ldots}+\underbrace{\int d \Phi_{R}\left|M_{R}(\ldots .,[g], \ldots .)\right|^{2}}_{-\frac{a}{\epsilon^{2}}-\frac{b}{\epsilon}+l+m \epsilon+n \epsilon^{2}+\ldots} \\
=\sigma_{\text {finite }}+\epsilon \sigma_{1}+\epsilon^{2} \sigma_{2}+\ldots
\end{gathered}
$$

where the scheme independent terms cancel and the analytic part of the expansion is scheme independent except for the first term. Regardless on the scheme the physical cross section should be obtained as

$$
\sigma=\lim _{\epsilon \rightarrow 0} \sigma^{D S}=\sigma_{\text {finite }}
$$

if the adopted regularization scheme is compatible with the unitarity of the theory, meaning with the statement of the the optical theorem. The purely $d$-dimensional treatment of all objects, the so called conventional (or naive) dimensional regularization is conceptually simpler, but it breaks supersymmetry and it gives ambiguities like $\operatorname{Tr}\left(\gamma_{5} \gamma^{\mu} \gamma^{v} \gamma^{\rho} \gamma^{\tau}\right)$ with chiral symmetries, but it is unitarity compatible [3]. However for the modern techniques of generalized unitarity [4] of the integrand reconstruction by gluing tree level helicity amplitudes, a four dimensional approach in general considerably simplifies the computations. In those cases the 4-dimensional treatment of the gluons is better compatible with supersymmetry and it is more amenable to helicity methods, where we glue amplitudes like the one coming from the Parke-Taylor [5] formula for Maximally Helicity Violating amplitudes

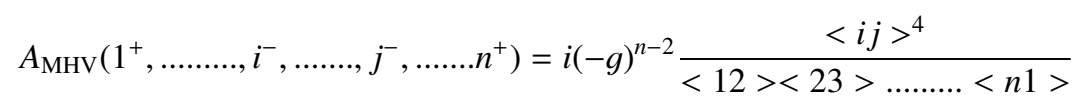

written here in terms of the helicity spinors, pedagogically introduced in [6]. The previous considerations imply the need for a dimensional reduction of the $d$ dimensional vector field to a four dimensional one together with a dimensional regularization of the loop integrals.

\section{Variants of the dimensional regularization}

There is an elegant way to unify essentially all common variants of dimensional regularization in a single framework, where all definitions can easily be formulated and where the differences and relations between the schemes become transparent [7]. Such a framework is based on the introduction of three vector spaces: the original four dimensional space $(4 S)$ and the infinite dimensional vector spaces, intrinsic in every dimensional scheme to provide the regularization of theories formulated in an arbitrary number of dimensions [8]. The space $(4 S)$ has metric tensor $g_{[4]}^{\mu \nu}$, then we have the "quasi- $d$-dimensional" space $\left(Q S_{[d]}\right)$ with metric 


\begin{tabular}{lcccc} 
& CDR & HV & FDH & DRED \\
\hline singular VF & $g_{[d]}^{\mu \nu}$ & $g_{[d]}^{\mu \nu}$ & $g_{\left[d_{s}\right]}^{\mu \nu}$ & $g_{\left[d_{s}\right]}^{\mu \nu}$ \\
regular VF & $g_{[d]}^{\mu \nu}$ & $g_{[4]}^{\mu \nu}$ & $g_{[4]}^{\mu \nu}$ & $g_{\left[d_{s}\right]}^{\mu \nu}$
\end{tabular}

Table 1. Treatment of vector fields in the four different regularization schemes, i.e. prescription which metric tensor has to be used in propagator numerators and polarization sums. The quantity $d_{s}$ is usually taken to be 4 .

tensor $g_{[d]}^{\mu \nu}$, the "quasi- $d_{s}$-dimensional" space $\left(Q S_{\left[d_{s}\right]}\right)$ with metric tensor $g_{\left[d_{s}\right]}^{\mu \nu}$. The "quasidimensionalities" of those infinite dimensional spaces are related by the following relations between the complex numbers:

$$
d_{s}=d+n_{\epsilon}=4-2 \epsilon+n_{\epsilon},
$$

implying the following formal direct sum and inclusions

$$
Q S_{\left[d_{s}\right]}=Q S_{[d]} \oplus Q S_{\left[n_{\epsilon}\right]} \quad S_{4} \subset Q S_{[d]} \subset Q S_{\left[d_{s}\right]} .
$$

Indeed since only gluons that appear inside a divergent loop or phase space integral need to be regularized, those are called "singular" gluons and all the others are called "regular". Based on that distinction the following variants of dimensional regularization and dimensional reduction are introduced. CDR ("Conventional dimensional regularization") in which singular and regular gluons (and other vector fields) are all treated as quasi- $d$-dimensional. HV ("'t Hooft Veltman") in which singular gluons are treated as quasi- $d$-dimensional but the regular ones are treated as 4-dimensional. For the dimensional reduction we have DRED ("original/old dimensional reduction"), where regular and singular gluons are all treated as quasi- $d_{s}$-dimensional, and FDH ("four-dimensional helicity scheme") so that singular gluons are treated as quasi- $d_{s}$-dimensional but external ones are treated as 4-dimensional.

The treatment of vector fields in the four different regularization schemes and the corresponding prescriptions on which metric tensor has to be used in propagator numerators and vectors polarization sums are summarized in the table 1 .

In order to use FDH and DRED, the variants based on the dimensional reduction, the crucial step is to split quasi- $d_{s}$-dimensional gluons into $d$-component gauge fields and $n_{\epsilon} \rightarrow$ $2 \epsilon$ scalar fields, so called $\epsilon$-scalars, as from the decomposition of the metric tensor

$$
\left(g_{\left[d_{s}\right]}\right)^{\mu v}=\left(g_{[d]}\right)^{\mu v}+\left(g_{\left[n_{\epsilon}\right]}\right)^{\mu v}
$$

with the algebra

$$
g_{\left[d_{s}\right]}^{\mu v}\left(g_{[d]}\right)_{v \rho}=\left(g_{[d]}\right)_{\rho}^{\mu}, \quad g_{\left[d_{s}\right]}^{\mu v}\left(g_{\left[n_{\epsilon}\right]}\right)_{v \rho}=\left(g_{\left[n_{\epsilon}\right]}\right)_{\rho}^{\mu}, \quad g_{[d]}^{\mu v}\left(g_{\left[n_{\epsilon}\right]}\right)_{v \rho}=0, \quad\left(g_{\left[n_{\epsilon}\right]}\right)_{v \rho} g_{[4]}^{\rho \mu}=0 .
$$

Since new degrees of freedom enter in the game, the independence of the gauge boson manifest in the couplings of the $\epsilon$-scalars resulting in different renormalization constants and $\beta$-functions. In QED the gauge coupling $e$ and the evanescent coupling $e_{e}$ renormalize differently being not protected by the Lorentz and the gauge invariance on $Q S_{[d]}$

$$
\beta_{e}=\mu_{\mathrm{DS}}^{2} \frac{d}{d \mu_{\mathrm{DS}}^{2}}\left(\frac{e}{4 \pi}\right)^{2}=\frac{4}{3}\left(\frac{e}{4 \pi}\right)^{4}+\ldots
$$




$$
\beta_{e_{e}}=\mu_{\mathrm{DS}}^{2} \frac{d}{d \mu_{\mathrm{DS}}^{2}}\left(\frac{e_{e}}{4 \pi}\right)^{2}=6\left(\frac{e}{4 \pi}\right)^{4}-6\left(\frac{e}{4 \pi}\right)^{2}\left(\frac{e_{e}}{4 \pi}\right)^{2}+\ldots
$$

Even by imposing the renormalization condition $e=e_{e}$ the flows of the two couplings are different [7].

\section{FDF:Four dimensional formulation of the FDH scheme}

FDF is a novel regularization approach that was introduced to reproduce FDH results at the one-loop level [9]. Starting from unregularized expressions in one loop diagram the loop momenta are shifted as $\ell_{[d]} \rightarrow \ell_{[4]}+i \mu \gamma_{5}$ before any other algebraic manipulation is performed. The matrix $\gamma_{5}$ provides the chirality in four dimensions. The scale $\mu$ corresponds to the other $d-4$ dimensional components of the loop momentum and serves as regulator for the in general divergent quasi- $d$ dimensional loop momentum in the integral. By definition, odd powers of $\mu$ are set to zero, resulting in the useful relation

$$
\ell_{[d]} \ell_{[d]}=\ell_{[d]}^{2}=\ell_{[4]}^{2}-\mu^{2} .
$$

One main advantage with FDF approach is that the Lorentz algebra is realized in strictly four dimensions. By construction the treatment of $\gamma_{5}$ in FDF unifies those of BreitenlohnerMaison and the anti-commuting chiral prescriptions like in the 't Hooft-Veltman [10].

Due to the previously declared shift the fermionic cutted lines in the diagrams obey to a generalized Dirac equation

$$
\left(\ell_{[4]}+i \mu \gamma_{5}+m\right) u(\ell)=0
$$

By expressing the four momenta in terms of the massless four-momenta $\ell_{[4]}^{b}$ and $q_{\ell[4]}$ the spinors generalized solutions are

$$
\left.\left.u_{+}(\ell)=\left|\ell_{[4]}^{b}\right\rangle+\frac{m-i \mu}{\left[\ell_{[4]}^{b}, q_{\ell[4]}\right]} \mid q_{\ell[4]}\right] \quad u_{-}(\ell)=\mid \ell_{[4]}^{b}\right]+\frac{m+i \mu}{<\ell_{[4]}^{b}, q_{\ell[4]}>}\left|q_{\ell[4]}\right\rangle
$$

with the usual notation of the helicity spinors. In [9] it is possible to find the wave functions for the cut gluonic and scalar lines for the consequent dimensional reduction involved in this procedure.

At one loop it is known the decomposition of any scattering amplitude in terms of a basis of master scalar integrals á la Passarino-Veltman [11]. By FDF we have a tool to add additional basis integrals at one loop consisting, in the case of having massless internal lines, into two sets of additional boxes $I_{4}\left[\left(\mu^{2}\right)^{2}\right], I_{4}\left[\mu^{2}\right]$, additional triangles $I_{3}\left[\mu^{2}\right]$ and additional bubbles $I_{2}\left[\mu^{2}\right]$, with the notations of having insertions of $\mu^{2}$ in the numerators of those additional master integrals. The decomposition of that amplitude in this basis involve coefficients of the additional integrals that are independent of the dimensional regulator, therefore those coefficients can be computed by integration contours using the methods of algebraic geometry [12]. The amplitude in now expressed in terms of larger set of integrals that in principle they could be evaluated as they are, however it is usually easier to evaluate them in terms of standard integrals. When we do that the dependence on the $\epsilon$ regulator reappears, in fact at one loop the conversion to the standard integrals amounts to a shift of the dimensionality, one has dimension shift identities like [15]

$$
I_{4}\left[\left(\mu^{2}\right)^{2}\right]=-\epsilon(1-\epsilon) 16 \pi^{2} I_{4}^{8-2 \epsilon}[1] .
$$

For a box integral we have now five degrees of freedom instead of four. The so called quadruple cut [13], which is the modern procedure of extracting the leading singularity of 
one-loop amplitudes (contrast it to [14]) and which allows the calculation of the box coefficients in the scattering amplitudes, is in terms of this basis a one-dimensional surface $\mu$-dependent more than a single point in the space of the momenta. However after gluing the corresponding amplitudes the box coefficients of the two integrals in the extended basis are still found by collecting in the numerators respectively the coefficients of the monomial $\left(\mu^{2}\right)^{2}$ and $\mu^{2}$. In fact by removing factors with $\epsilon$ exponents from the measure $d^{4-2 \epsilon} k=d^{4} k d^{-2 \epsilon} k$ and multiplying by a weight function of $\mu^{2}$ to isolate different integrals we find in terms of the block amplitudes the coefficients of the extra basis integrals as

$$
c_{i}=\oint \frac{d \mu^{2}}{\mu^{2}} A_{\text {tree }}^{1}\left(\mu^{2}\right) A_{\text {tree }}^{2}\left(\mu^{2}\right) A_{\text {tree }}^{3}\left(\mu^{2}\right) A_{\text {tree }}^{4}\left(\mu^{2}\right),
$$

with a final result compatible with [16].

\section{Foundational work to perform two loops reductions in FDF}

First of all we need a basis, this has been provided in pure 4 dimensions for planar diagrams in [17]. The notations for the double box $P_{n_{1}, n_{2}}^{*}$, refer to $n_{1}$ lines on the right and $n_{2}$ lines on the left of the double box. In this basis we have two integrals for massless, one-mass, diagonal-two mass double box $P_{2,2}^{*}$. Three integrals for short side two mass, three-mass double box. Four integrals for four masses double box. One integral for massless pentabox $P_{3,2}^{*}$. A pure four dimensional basis is reduced to this with master integrals containing up to height propagators. More complicated integrals can be reduced to them up to $\epsilon$-terms. The new feature compared with the one-loop case is the presence of irreducible numerators even when it is simply involved the scalar product of a loop momentum with an external leg, meaning that they cannot be written as linear combination of the denominators propagators and external invariants.

To perform the reduction to the previous basis at two loops the tensor reduction is still necessary and useful but it is not sufficient, because some additional technology is needed in order to take care of the irreducible invariants and their powers. That technology is based on the so-called integration by part identities (IBP) [18]. This technology is based on an axiom of dimensional regularization that the integral of a total divergence is zero, because there are no boundary terms

$$
0=\int d^{d} \ell_{1} d^{d} \ell_{2} \frac{\partial}{\partial \ell_{1}^{\mu}} \frac{v^{\mu}}{\text { Denominator }}
$$

this gives linear relations between integrals that can be solved in terms of the independent master integrals. In the notation of the previous equation a so called IBP generating vector is included which takes care of the fact that no doubled propagators are generated in the IBP, this simplifies the solution of the system of the IBP. By identifying the IBP generating vectors would provide a quicker approach to solve the system for generating master integrals [17]. By the definition of the vectors $v^{\mu}$, they are algebraic geometric objects arising from appropriate polynomial divisions, their algebraically geometric properties as well as their connection to the different topologies of the basis need still to be completely studied. In the aim of finding a two-loop basis with coefficients still independent of the regularization parameter, the IBP arise a kind of line-one problem:

$$
\frac{\partial}{\partial \ell_{1}^{\mu}} \frac{\ell_{1}^{\mu}}{\text { Denominator }}=\frac{4-2 \epsilon}{\text { Denominator }}+\ldots
$$


consequently $\epsilon$ seems to be intrinsic to these reductions bringing to a decomposition of the type

$$
\mathrm{Ampl}=\sum_{j \in \text { Basis }} c_{j}(\epsilon) \mathrm{Int}_{j}
$$

as for instance in the one-loop bubble by Passarino-Veltman reduction

$$
B^{\mu v}\left(k^{2}\right)=\frac{2-\epsilon}{4(3-2 \epsilon)} B_{0}\left(k^{2}\right) k_{[4]}^{\mu} k_{[4]}^{v}-\frac{1}{4(3-2 \epsilon)} B_{0}\left(k^{2}\right) g_{[4]}^{\mu \nu} .
$$

This is a problem in view of implementing the $4-2 \epsilon$ dimensional generalized unitarity program, for which it is desired a basis with coefficients which are $\epsilon$ independent. The regularizer $\epsilon$ is indeed something deeply related to the integration on the real slice. By the methods of generalized unitarity we want indeed to perform integration on contours in the complex plane to isolate the appropriate residues, clearly we cannot have a basis which is $\epsilon$ dependent, because the $\epsilon$ dependence is obtained from integration on the real slice. The conjecture considered here [19] is that again at two-loop there is an augmented basis, which now will depend on $\mu_{1}^{2}, \mu_{2}^{2}, \mu_{1} \cdot \mu_{2}$. The tree amplitudes to be glued in the integrand reconstruction indeed come from the six dimensional extension of the theory [20]. By FDF we can reconstruct the total dependence of the two-loop integrands in terms of the extra dimensions using an appropriate modifications of FDF in terms of six dimensional ingredients [21]. However the recursive construction of the Dirac gamma matrices in higher dimensions is based on an operation of tensor product having the structure with four dimensional ingredients as seed of generation of the Dirac representation of the Lorentz group in higher dimensions [22]. In this sense FDF seems to be a basic block so that modulo multiplication with appropriate sigma Pauli matrices, the FDF results for the tree amplitudes computations [9] in the generalized unitarity procedure can be exported to two loops.

The way proposed here to reach such an augmented basis is thorough four-dimensional IBP

$$
0=\int d^{d} \ell_{1} d^{d} \ell_{2} \frac{\partial}{\partial \ell_{[4]_{i}}^{\mu}} \frac{v_{[4]}^{\mu}}{\text { Denominator }}
$$

The involved IBP generating vectors $v_{[4]}^{\mu}$ will be dependent on the $\mu_{1}^{2}, \mu_{2}^{2}, \mu_{1} \cdot \mu_{2}$ and even for simple integrals like the double box they have not been computed yet, however it is expected that their algebraic geometry is very different form the ones coming from the IBP in $d$ dimensions.

The last ingredient that we need is how to convert back to standard integrals. We have the additional components $\mu_{i}$ inside integrand we want to go back to recover $\epsilon$. There are basically two techniques to be combined:

- take a Gram determinants $\left[G\left(\left\{p_{i}\right\},\left\{q_{i}\right\}\right)\right]=\operatorname{det}_{i, j}\left(p_{i} \cdot q_{j}\right)$, do Feynman parametrization to get relations like this

$$
P_{2,2}^{*, *}\left[\left(\mu_{1}^{2}\right)^{2}\right]=-\frac{\epsilon(1-\epsilon)}{(3-2 \epsilon)(1-2 \epsilon) G_{124}^{2}} P_{2,2}^{*, *}\left[G^{2}\left(\ell_{1}, 1,2,4\right)\right]
$$

where together with a rational function of $\epsilon$ there is the dependence on $G_{124}^{2}$, an invariant of the external momenta;

- perform standard (d-dimensional) IBPs with $\mu_{i}$ factors and irreducible products inserted in the numerators

$$
0=\int d^{d} \ell_{1} d^{d} \ell_{2} \frac{\partial}{\partial \ell_{i[d]}^{\mu}} \frac{\left\{1, \mu_{1}^{2}, \ldots . .\right\}(\text { irreducible })^{j} v_{[4]}^{\mu}}{\text { Denominator }} .
$$




\section{Conclusions}

Alternative dimensional regularization schemes are available for higher order computations in perturbative gauge theories. However there is not a wide use of them. It is needed to provide more practical examples to show the efficiency of those schemes. The four dimensional formulation (FDF) of the four dimensional helicity scheme (FDH) is a proposal to get the cut constructible and the rational part of one-loop amplitudes by just four dimensional cuts. FDF is efficient to find contributions of evanescent operators in perturbative computations. The foundations for $d$-dimensional unitarity within FDF at two loops have been discussed.

\section{References}

[1] G. Leibbrandt, Rev.Mod.Phys. 47, 849 (1975) .

[2] D. Bardin, G.Passarino, The Standard Model in the making ( Claredon Press, New York, 1999 ) pp. 166-169.

[3] G. 't Hooft, M.J.G. Veltman, Nucl.Phys. B50, 318 (1972).

[4] Z. Bern, L.J. Dixon, D.A. Kosower, Annals Phys. 322, 1587 (2007).

[5] I suggest for a review: T.R. Taylor, Phys.Rept. 691, 1 (2017).

[6] M.E. Peskin, arXiv:1101.2414 [hep-ph].

[7] C. Gnendinger et al., Eur. Phys. J. C77, 471 (2017).

[8] J.C. Collins, Renormalization (Cambridge University Press, Cambridge, 1984) pp. 6287.

[9] A.R. Fazio, P. Mastrolia, E. Mirabella, W.J. Torres Bobadilla, Eur. Phys. J. C74, 3197 (2014).

[10] C. Gnendiger, A. Signer, Phys.Rev. D97, 096006 (2018).

[11] G. Passarino, M. Veltman, Nucl. Phys. B160 151 (1979).

[12] D. A. Kosower, K. J. Larsen, Phys.Rev. D85, 045017 (2012).

[13] R. Britto, F. Cachazo, B. Feng, Nucl.Phys. B725 275 (2005).

[14] Eden, Landshoff, Olive, Polkinghorne The analytic S-matrix (Cambridge at University press, Cambrdge, 1966) chap. 2.

[15] Z. Bern, A.G. Morgan, Nucl.Phys. B467, 479 (1996).

[16] S.D. Badger, JHEP 0901, 049 (2009).

[17] J.Gluza, K. Kajida, D. A. Kosower, Phys.Rev. D83, 045012 (2011).

[18] For a review: V.A. Smirnov, Analytic tools for Feynman integrals (Springer, London, 2012 ) pp. 127-154.

[19] D.A. Kosower, Maximal Unitarity and Algebraic Geometry, talk given at IAS focused program in Scattering Amplitudes in Hong Kong, November 2014.

[20] S. Badger, C. Bronnum-Hansen, H. Bayu Hartanto, T. Peraro, Phys.Rev.Lett. 120 no.9, 092001 (2018).

[21] A.R. Fazio, P. Mastrolia, W.J. Torres, to appear.

[22] P. van Nieuwenhuizen, Six Lectures at the Trieste 1981 Summer School on Supergravity, in Supergravity 81 ( Cambridge University Press, Cambridge, 1982) p. 151. 